\title{
Psychological Cognition and Thinking Needs in Visual Communication Design
}

\author{
Dandan Zhao ${ }^{1, a}$, Bo $\operatorname{Pan}^{2, b}$ \\ ${ }^{1}$ Wuhan University of Technology Shenyang Jianzhu University China \\ ${ }^{2}$ Shenyang Jianzhu University China
}

\begin{abstract}
The visual information design refers to the communication and exchange with the real world through the graphic signification. The understanding of a graph is a visual recognition process of the graphic object. The audience can feel the intangibility of the design work behind the tangibility during the transmission, perception, communication and resonance processes. The unconscious mind of the audience is aroused under the visual impact, thus reaching the goal of transmitting the concept of information appeal. Influenced by the holistic view of the Gestalt Psychology, the modern cognitive psychology highlights the comprehensive analysis of human cognitive process, while Gestalt psychologists lay the emphasis on the integrity of experience and behavior. According to the principle of Gestalt Psychology, the form perceived is not the direct imitation of an objective thing, but instead, it is the perceptual construction activity when eyes capture the thing. As a manifestation system in the visual perception research field, the Gestalt Psychology teases the related perceptual organization principles for the human cognitive process and promotes the development of the visual perception researches. Based on the Gestalt Psychology, it is proposed in this research to study psychological phenomena from the holistic dynamic structure, explore the visual information design, and exploit the design field of view and creative thinking, in an effort to form a new design philosophy which will play an important role in improving the aesthetic effect and visual impact of the design work.
\end{abstract}

\section{Symbolic Language and Psychological Cognition of Visual Information Design}

Based on human physiological, psychological and sociocultural needs, the visual information design embodies the harmonious relationships between man and society, man and nature, and man and man. To be more specific, it realizes the communication and exchange with the real world through the symbolic language of a graph, and the audience's understanding of this graph is rightly the recognition process of the graphic object. The information spreading and communication mainly rely upon the visual language communication with the audience. As an objective existence of human percipience, the visual language transmits the specific information contents via the visual symbols and symbol system, and it is produced by integrating the physical attribute and spiritual attribute in a special way. The combination of different visual morphological structures in the visual design aims to create an orderly structure, which accords with the regular art language. The representation and reasonability of the order are core factors for creating such structural form.

As for the image, text, color, spatial organization, etc., which are involved in the information design, particular attention is paid to the preciseness of the structural forms as well as the morphological visuality and aesthetic requirements. We live in a world of symbols, and our internal experience is stimulated by the internal drive of ideographic symbol marks. The expression and mastery of human consciousness relies upon a symbol system. As said by the Japanese linguist Yoshihiko Ikegami in An Introduction to Semiotics, "when a thing represents another thing as its substitute, the function of the thing is called symbolic function, and thing undertaking this function is called symbol. In short, the function of symbol is substitution, giving something some meaning and extracting some meaning from something". (1) That a graphic symbol is transformed into a conveyable implicit structural graph embodies the prime power of human life and the influence of deeper psychological factors. Moreover, this stimulates the agreement with the thoughts and emotions of the audience, stresses the isomorphism of the object entirety and psychological entirety, and conveys intense impact force to arouse the internal touch of the audience. In consideration of the receptive psychology of the audience, they perceive the intangibility of the design work behind the tangibility during the transmission, perception, communication and resonance processes. With visual impact, their unconscious mind is aroused so as to reach the goal of transmitting the concept of information appeal. Nonverbal communication is manifested in the information transmission process through different 
symbolic languages. In Visual Language, Corbins systematically investigated the visual elements and their combination laws, and analyzed the modeling, image and composition from the perspective of psychology. As a general rule, the concept of symbol is closely related to human life and natural environment, and it derives from human visual experience. It is an abstract method to utilize point, line, plane, color, space and sense of form. For instance, sun is felt like a dot, straight line is upright and powerful just like pine tree, curve is as soft and warm as cloud, square gives us the honest and sincere image, and triangle makes us fell stable, etc. The emotions conveyed by the above all are abstract and general. The understanding and recognition of graphic symbols vary from person to person due to their differences in association or feeling, so designers should use different visual symbolic languages to express their design thoughts in consideration of different service objects.

To pay attention, one needs to perceive firstly. The perceived may be memorized or forgotten, which should be considered by the designers, and man will selectively forget some things with the time after a sensual experience.

\section{Visual Perception and Gestalt Principle in Visual Information Design}

The perception in the visual design follows its own basic laws. The relationship between perceptual experience and cognitive structure has been a research hotspot in the fields of cognitive science and linguistics. Psychologists have generated very excellent artistic effects using the principle of visual perception in their research design. The Gestalt principle concretely explains how the visual system perceives the world. The form perceived is not the direct imitation of an objective thing, but instead, it is the perceptual construction activity when eyes capture the thing. In essence, the visual design is a visual perception activity restricted by visual thinking mode. Form is the product of instant organization generating visual perception, and it is an entirety formed by perceptual organization. Not being equal to the sum of constitutive elements, the entirety can be clearly separated from other objects in the background. Just like feeling, perception is generated when an object directly acts upon sensory organ, and without this direct action, there will be neither feeling nor perception. According to the sensory characteristics, the perception is divided into visual perception, auditory perception, tactile perception, etc. The size, shape and distance of an object or graph belong to visual perception, and the perception of direction, rhythm and tempo of sound belong to auditory perception. It can be further divided into spatial perception and temporal perception based on the object characteristics reflected by human brain, where the spatial perception reflects the size, shape and distance of the object, and the temporal perception reflects the continuity and sequence of the object. The special form in perception is illusion, and the principle of visual illusion tells us how man observes. Visual sense is the head of all feelings. A half of the resources in human brain are used to receive and interpret what the eyes see, and what is really used to observe is indeed brain. The human cognitive system will differentiate figure-ground elements and give more attention and memory. Discovered by the famous Italian psychologist Gaetano Kanizsa, this unique illusion is called Kanizsa Triangle, and one of the earliest visual illusion is Müller-Lyer illusion, both of which are convincing proofs for visual illusion. (2)

According to the theory of Gestalt Psychology, a visual tension, which arouses attention from the audience, exists in the graphic form of information design, and this is the visual force of form and a reaction generated by brain. Rudolf Arnheim has once pointed out that an external acting force intrudes into the organism, which is involved in the perceptual activity and breaks through the overall equilibrium of the nervous system. We cannot image the stimulus as very gently printing a static style onto a stubbornly resisting medium for sudden puncturing. But instead, it is actually a battle, the impact caused by the intruding forces is resisted by physiological forces, which throw themselves into the battle against the intruding force or transform these intruding forces into simple styles. In this way, the product generated by the mutual contest of two opposed forces is the finally generated object of perception. In the visual design, the impact force is actually like this intense feeling of "sudden puncturing", that is, the "tendentious tension" said by Arnheim.(3)

As a language of design form, the visual information design has unique propagandizing, enlightening, guiding and communicating functions in modern social life. How to realize the perfect combination of the functions of this artistic form with visualized expression is what is cared and investigated by designers. The information design is implemented by a designer who transmits the information expressed by the text images and symbols and various signs he/she sees to the audience. Efforts should be made by graphic creative thinking to deeply investigate the Gestalt theory of cognitive psychology and formal characteristics of visual thinking, and thus new vigor will be endowed to graphic objects with the development of the times. The research results obtained by the school of Gestalt Psychology regarding the graphic visual perception have not only laid a theoretical foundation for the manifestation of formal beauty in the visual design but also will motivate our creative thinking potentials so that the language expression of this form becomes richer and more colorful. When it comes to the meaning of information, it not only needs to accord with the requirements of the audience but it is also the precondition for the understanding of perception. Only meaningful information can be perceived and motivate visual movement. For example, the sign expressing the information of a danger arouses the vigilance of literate people, but it will just stimulate a gaze for illiterate people. However, the graphic symbolic language will contribute to the cognition through color and shape. As the rule of visual movement is applied to the visual design, the selection of the optimal field of vision, the arrangement of the elements inducing the visual flow direction, and the visual process planning constitute a complete visual movement process. According to the researches carried out by Gestalt psychologists, the attention of a man is 
unbalanced if his/her vision is limited within a specific scope. The different feelings given by different fields of vision over the psychological hints have a bearing on the attention and visual recognition. The process design is a visual communication process and a perception process. The feeling at first glance drives the audience to be attracted and touched by the graph, the excitement and pleasure at the second glance guide the attention of the audience to the content theme, and finally these feelings are left in their memory. The visual process is designed rightly based on this psychological process. By virtue of the graphs and texts containing all kinds of information, the optimal visual language is used to transmit the meaningful information to the user in the information design. The functional, economic and aesthetic values of the information design depend on the information transmission to a great extent. A good design should not just satisfy the requirement for the visual impact or just give visual physiological and psychological comfort and enjoyment of beauty, but should aim to realize the enjoyment function of the meaningful information.

A group of experimental comparisons were conducted according to visual perception. The snacks competitively purchased by the customers were selected and divided into three groups, where there was only literal expression in the first group, product photos were included in the second group, and physical products existed in the third group. According to the experimental results, the physical products showing the more intuitive object image enjoyed the highest bidding price as shown in the following figure:

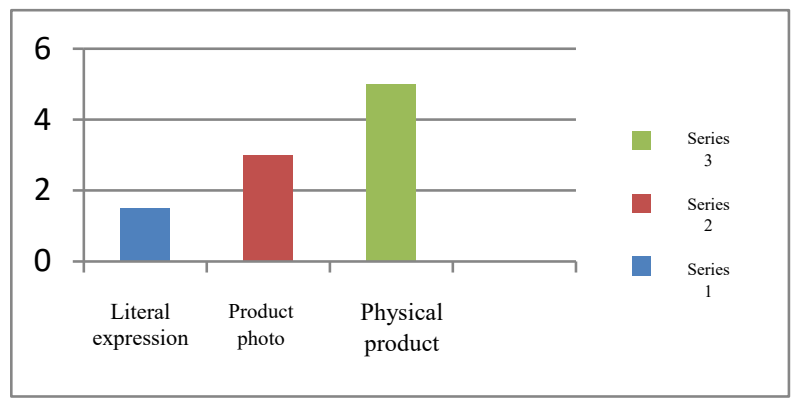

\section{Thinking Needs and Innovation of Visual Information Design}

The visual information design aims to study the thinking activity of the audience, and any scientific research will unavoidably belong to the category of psychology and may involve other fields. Human thinking is a complex mental activity of human when solving a problem. To understand the human thinking, we need to cognize their needs. The needs of human, which is an intelligent creature, are much more complicated than those of other species. Reflecting the psychological needs and social needs by human brain, human needs constitute the internal impetus of individual behaviors and psychological activities, and play an important role in human activity, psychological process and characteristic expression. Human needs can lead to the emergence of brand-new products or new functional breakthroughs. A theory of human needs structure was proposed by the American psychologist Maslow in his thesis titled $A$ Theory of
Human Motivation Psychological Review in 1943, where the human needs were divided into five levels: physiological needs, safety needs, emotional and affiliation needs, esteem needs and self-actualization needs. Maslow believed that the five needs presented a step-by-step rising trend in regular sequence. According to his hierarchy of needs, human needs were mutually associated and overlapped with interdependence. The selfactualization need would appear only after the other needs were satisfied in succession. Maslow's theoretical investigation only aimed at the achievers in social undertakings, so it could not scientifically represent the hierarchy of needs of the whole social group, but his research could still be used for reference in the researches on design psychology. Following Maslow, Richard Gerrig and Philip Zimbardo put forward the needs of expressing power, conquering and attacking in Psychology and Life, the incentive psychologist Alderfer supplemented the hierarchy of needs inIn Empirical Test of New Theory of Human Needsin 1969, and the human needs were divided into: existence needs, relatedness needs and growth needs. (4) The needs of the audience is inseparable from the design, and the innovative design can be completed through the user needs.

With the rapid development of network and digital technology, a large number of new lifestyles are changing our life. The traditional shopping mode and online shopping mode not only change our life but also drive the national economy. Facing the development of intelligent e-commerce and the brand-new concept of internet market, the designers should put more efforts into the innovative design, pay more attention to the combination of psychology with the Gestalt method and principles of aesthetics, explore into the internal relationship between perception and visual image, discover and master the design and application skills of visual information design elements, so as to improve their own visual design capability. The principle of design psychology unconsciously plays an immeasurable role in the modern design practice, and will continue to serve as the theoretical basis for and exert an important influence on the future design practice of the designers. As the development of the design field is deepened, the design concept and objective are clear-cut. Hence, the designers should not only produce good design works but also effectively utilize the psychological knowledge to realize the deeper and innovative application of the researches on the design theory and design practice.

\section{Annotation}

1. Advertising Visual Language written by $\mathrm{Zhu}$ Jianqiang, Xiamen: Xiamen University Press, Page 6, 2000.

2. Every Designer Needs to Know about People written by (British) Susan Weinschenk and translated by $\mathrm{Xu}$ Jia/Ma Di/Yu Yingyi, Beijing: Posts \& Telecom Press, Page 2, 2013.

3. Advertising Visual Language written by Zhu Jianqiang, Xiamen: Xiamen University Press, Page 66,2000 . 
4. Design Psychology written by Dai Linong, Beijing: China Forestry Publishing House, Page 66-69, 2014.

\section{REFERENCES}

1. The Image and the Eye-Further Studies in the Psychology of Pictorial Representation [M] written by (British) E.H.Gombrich and translated by Fan Jingzhong, Yang Siliang, $\mathrm{Xu}$ Yiwei, Lao Chenglie, Zhejiang: Zhejiang Photography Publishing House, 1989

2. The Sensor of Order [M] written by [British] Gombrich and translated by Fan Jingzhou, Yang Siliang, $\mathrm{Xu}$ Yiwei, Hunan: Hunan Science \& Technology press, 2003.

3. Advertising Visual Language [M] written by Zhu Jianqiang, Xiamen: Xiamen University Press, 2000.

4. Design Psychology written by Dai Linong, Beijing: China Forestry Publishing House, 2014.

5. Emotional Design [M] written by Donald. A. Norman and translated by $\mathrm{He}$ Xiaomei and OuQiuxing, Beijing: CITIC Press, 2019.

6. The Magic of Design $[\mathrm{M}]$ written by Deepa Prahalad/Ravi Sawhney and translated by Liu Qianqian, Han Fang, Hu Chuqiao, He Yunchao, Beijing: China Renmin University Press, 2014.

7. Universal Principles of Design written by William Lidwell/Kritina Holden/Jill Butler and translated by Yi Mo/Liu Zhuangli, Liaoning: Liaoning Science and Technology Publishing House, 2018.

8. Every Designer Needs to Know about People written by (British) Susan Weinschenk and translated by Xu Jia/Ma Di/Yu Yingyi, Beijing: Posts \& Telecom Press, 2013. 